\title{
Food as a contextual cue in counterconditioning experiments: Is there a counterconditioning process?
}

\author{
ELIZABETH D. CAPALDI, DONNA M. VIVEIROS, and DAVID H. CAMPBELL \\ Purdue University, West Lafayette, Indiana
}

\begin{abstract}
In three experiments, counterconditioning was found to reduce fear less effectively than extinction. In Experiments 1 and 2, the resigtance to extinction of avoidance was greater if food was given during extinction of fear to the CS than if no food was given, even when exposure to the CS and numbers of food and no food confinement trials were equated. It is sug gested that these results could be attributed to contextual control of fear extinction by the food cue and/or to frustration produced by removing food for the counterconditioning group. Experiment 3 also found counterconditioning to be less effective than extinction and provided evidence that this difference occurs because of contextual control of fear extinction by the food cue. Measuring conditioned suppression of licking, in a test with no food present, less fear was shown if no food had been present during fear extinction, and greater fear was shown if no food had been present during fear conditioning. These results indicate that food is an important part of the context controlling fear and fear extinction. It is suggested that there may be no unique counterconditioning process. Rather, when counterconditioning procedures are employed, rules governing interference paradigms in general may apply. Thus, in a test for fear following counterconditioning, fear will be shown to the extent the test situation is similar to that in which fear conditioning occurred rather than that in which fear reduction occurred.
\end{abstract}

In her frequently cited paper, Mary Cover Jones (1924) described methods of eliminating inappropriate fears in children. Her "method of direct conditioning" consisted of associating the feared object with a situation "capable of arousing a positive (pleasant) reaction." For example, to eliminate fear of rabbits in a child named Peter, she placed a caged rabbit a few feet from Peter while he was eating candy. This procedure is now referred to as counterconditioning. An early example of counterconditioning in animals is Pavlov's (1927) description of a dog that became excited at being placed in a restraining stand, but gradually became quieter when it received all its food in the restraining stand. The behavior therapy of systematic desensitization is based on counterconditioning phenomena, and it is highly successful (e.g., Kazdin \& Wilson, 1978). Despite this background, well-controlled experiments to demonstrate counterconditioning effects in animals have been largely unsuccessful.

Reviews of counterconditioning experiments (Dickinson \& Pearce, 1977; Reid, 1973; Wilson \& Davison, 1971) indicate that, when exposure to the

This research was supported in part by the National Institute of Mental Health, Grant MH34119, to the first author. Reprints can be obtained from the first author, Department of Psychological Sciences, Purdue University, West Lafayette, Indiana 47907. feared cue is equated, most comparisons of counterconditioning and extinction have not found that counterconditioning reduces fear any better than does mere exposure to the feared cue. Two exceptions are one study by Poppen (1970) and another by Wilson and Dinsmoor (1970).

Poppen (1970) reported that counterconditioning was more effective than extinction when using a conditioned suppression procedure. With this procedure, a conditioned stimulus (CS) is established as a signal for noncontingent shock while the animal is barpressing for food. The measure of fear is suppression of barpressing. Counterconditioning in this situation is accomplished by delivering free food during a CS which has previously been established as a cue for shock. There are two problems with this procedure. First, pairing a CS noncontingently with food while an animal barpresses for food also suppresses responding (positive conditioned suppression, Azrin \& Hake, 1969). So, if extinction of fear is successful with a counterconditioning procedure in this situation, animals may still suppress responding to the CS. Alternatively, response suppression could reflect continued fear. So the response suppression measure is ambiguous. Second, competing peripheral responses could account for any pattern of results in this situation. That is, the counterconditioning group makes a response to the food received in this situation, and this response could 
facilitate or inhibit barpressing, making the interpretation of results difficult. These considerations may explain why results using this procedure have been contradictory (Delprato \& Jackson, 1973; Poppen, 1970). Also, as pointed out by Dickinson and Pearce (1977), the only experiment using this procedure that obtained a difference favoring the counterconditioning group (Poppen, 1970) changed the schedule for the baseline response from VI $60 \mathrm{sec}$ in acquisition to consistent reward in test for the counterconditioning group, which could account for the difference between this group and the control group.

Wilson and Dinsmoor (1970) trained rats to remain on a platform to avoid shock. The counterconditioning group was then fed on the platform and the test measured resistance to extinction in terms of time to leave the platform. The counterconditioning group left the platform more rapidly than a group put on the platform for the same amount of time as the counterconditioning group, but without food. This result is difficult to interpret in terms of competing responses. If anything, the counterconditioning group should have been conditioned to remain on the platform. However, there are two other processes that could affect behavior in this situation and in other counterconditioning experiments as well: frustration and stimulus change. As suggested by Dickinson and Pearce (1977), frustration may have occurred in the counterconditioning group, causing them to leave the platform sooner than the other group. The fed group was given food in the test, although how much was given was not stated. It is possible that the animals ran out of food, which produced frustration, and then escaped from the frustration (the test could last up to $21 \mathrm{~h}$ ).

Frustration could also affect behavior in counterconditioning studies that use the active avoidance situation. In these studies (e.g., Delprato \& Jackson, 1973; Klein, 1969; Nelson, 1966; Sermat \& Shephard, 1959; Wilson, 1973), training consists of active escape or avoidance learning using a stimulus as a signal for an aversive event $\left(\mathrm{CS}^{+} \mathrm{Av}^{\mathrm{V}}\right)$, usually shock. Subsequently, the CS+AV is paired with food for the counterconditioning group. A control group receives exposure to the $\mathrm{CS}^{+} \mathrm{AV}$ with no food. Resistance to extinction of the escape or avoidance response is then used as a measure of residual fear to the CS. Feeding the counterconditioning group during exposure and then testing it without food could result in frustration (Amsel, 1958), and frustration could produce responses motivated by fear (e.g., Brown \& Wagner, 1964; McAllister \& McAllister, 1971) or directly produce an escapefrom-frustration response (e.g., Daly, 1974). This would tend to increase resistance to extinction more in the counterconditioning group than in the exposedonly group.

The second process that could operate in a counterconditioning study is stimulus change. In counterconditioning experiments, the fed group experiences a large stimulus change between the counterconditioning phase and the test phase. Food is removed. In most experiments, the counterconditioned group has food continuously present during the counterconditioning phase. Thus, food can be viewed as a contextual or background cue. Recently, Bouton and Bolles (1979) showed that extinction is, to a large extent, specific to the context in which extinction occurs. Bouton and Bolles found renewed suppression to a CS following extinction when testing was given in a context different from that used for extinction. A counterconditioned group is tested in a situation different from that used for extinction-food is present during fear extinction but not during fear testing. This may explain why fed groups are sometimes more resistant to extinction than nonfed groups (e.g., Wilson, 1973). In Wilson and Dinsmoor's (1970) study, stimulus change could also have influenced the results. No food was given either group in training, then food was given to the counterconditioning group, but not to the exposedonly group, in the counterconditioning and test phases. Because no food was given in training, the context in test was more similar to that present in training for the nonfed group, which could account for their greater fear. The purpose of Experiments 1 and 2 was to determine if frustration and/or stimulus change influenced results in the active one-way avoidance situation, the situation most commonly used to investigate counterconditioning.

\section{EXPERIMENT 1}

Experiment 1 closely followed the procedures used by Wilson (1973). All groups were trained in an active one-way avoidance task in Phase 1 and were tested for resistance to extinction of this response in Phase 3. In Phase 2, all groups were confined to the shock side. Group R was fed in the presence of the $C S^{+A V}$ used during avoidance training. Group $\mathbf{N}$ had the same exposure to $\mathrm{CS}^{+\mathrm{AV}}$, but no food was given. These were the counterconditioning (Group R) and exposed-only (Group $N$ ) groups. The third group was a " partial-counterconditioning" group (Group P), which received food on half of its CS exposures and no food on the other half. Group $P$ thus experienced the $C S^{+A V}$ without food, minimizing the effects of changing context and frustration when the CS ${ }^{+A V}$ is received without food in the Phase 3 test. Nelson (1966, Experiment 1) gave one of his groups both food trials and confinement trials. However, the three confinement trials 
followed three food trials in four of the five replications, and the aim was to extinguish the counterconditioning effect. In the present experiment, food and nonfood trials were intermixed. If either frustration or context change, or both, have increased resistance to extinction in previous counterconditioning groups, Group P should be less resistant to extinction than Group R.

\section{Method}

Subjects. Thirty naive male albino rats, 77 days old on arrival from the Holtzman Co., Madison, Wisconsin, were the subjects.

Apparatus. The apparatus was a Lafayette Instrument Co. shuttlebox (Model 85103), consisting of two stainless steel modular testing units $(20.32 \mathrm{~cm}$ wide $\times 30.48 \mathrm{~cm}$ long $\times 19.69 \mathrm{~cm}$ high), each having an 18-bar grid floor (.4-cm diam) and white Plexiglas lid. The top portion of one wall in each unit consisted of a 9-cm-wide one-way mirror running the length of the box. The two units were connected by a common end panel with a 9.53-cm square opening located centrally at the bottom of the panels. An electronically controlled guillotine door on this opening controlled access between compartments. An inoperative light was located $13.5 \mathrm{~cm}$ above the grid floor at the opposite end of one unit (startbox). Microswitches connected to the counterbalanced grid floors monitored subject movement between compartments. Scrambled shock was delivered to the grid floor on one side of the shuttlebox by a Lafayette Instrument Co. Master Shocker (No. 82404). Tones were delivered using a 2.9kHz Mallory Sonalert (No. SC608), in series with a 3,300-ke resistor, located just outside the startbox end panel $14.0 \mathrm{~cm}$ above the level of the grid floor. The tone intensity was $63 \mathrm{~dB}$, against a background of $50 \mathrm{~dB}$. Light was provided in both compartments by two 6-W bulbs above the white Plexiglas lid. Times were recorded automatically by a Cronus digital stopclock interfaced with the computer.

Procedure. The experiment was run by two experimenters, each of whom ran half the subjects in each group. After 10 days of ad-lib feeding, rats were assigned randomly to the three groups, $R, N$, and $P$, and all rats were handled for $1 \mathrm{~min}$. After the second day of handling, all food was removed from the cages of all rats (Day 1) and a 14-g/day (Wayne Lab Blox) deprivation schedule began.

Phase 1 began on Day 1. Each rat received five one-way avoidance trials daily until a criterion of 10 successive avoidance trials (latency less than $5 \mathrm{sec}$ ) was reached. Once a rat had met its criterion, it was handled briefly when its turn in the running order occurred. On avoidance trials, the rat was placed in the shock compartment. Twenty seconds later, the tone CS came on, the door to the safe compartment was raised, and the clock started. Five seconds later, a .5-mA .5-sec shock US was delivered to the grids of the shock compartment. All stimuli remained on until either a correct response occurred or $60 \mathrm{sec}$ had elapsed, at which time the door closed and the stimuli and the clock stopped. No rats stayed in the shock compartment for the 60-sec maximum, hence all stimuli on all trials were response terminated. Once a response was made, the rat remained in the safe compartment for $30 \mathrm{sec}$ before it was removed and replaced in the shock compartment for the next trial. Response latency was recorded (to the nearest $.01 \mathrm{sec}$ ). Rats were run in squads of three, with one rat from each group. Each rat within a squad was brought into the room singly in a holding cage for its trials. Running order within a squad was determined randomly daily with Squad 1 always preceding Squad 2, etc. Each squad was fed its daily ration $15 \mathrm{~min}$ after completion of its trials. There was a 2-day break between Days 1 and 2 because of equipment failure. The last rat finished Phase 1 in 12 days. After an animal finished its trials, it was picked up and replaced in its cage when its turn in the sequence came. If all three rats in a squad reached criterion, all three were handled in turn. In Group $\mathbf{R}$, trials to criterion ranged from 16 to 41 , in Group $N$ they ranged from 18 to 44 , and in Group P from 16 to 51 .

On the last 2 days of Phase 1 , each rat received $20.045-\mathrm{g}$ Noyes pellets in a Mason jar lid attached to the floor of its home cage $10 \mathrm{~min}$ prior to receiving its daily ration (adjusted to compensate for the pellets to total $14 \mathrm{~g}$ per day consumed for each rat). If a rat ate the pellets within the $10 \mathrm{~min}$, the lid was removed from the cage. If a rat did not eat all the pellets immediately, the lid remained in the cage overnight.

Phase 2 started the day after Phase 1 ended for the last rat and lasted 8 days. Each rat received six trials daily with no response possible. On a trial, the rat was placed in the shock compartment and $20 \mathrm{sec}$ later the tone was sounded for $5 \mathrm{sec}$. Ten seconds later, the rat was removed and placed in a holding cage for $20 \mathrm{sec}$ between trials. Group $\mathbf{R}$ was placed in the compartment facing a jar lid containing $30.045-\mathrm{g}$ Noyes pellets on all trials. The jar lid was in the rear left corner of the compartment (the corner away from the door). Because the food was present throughout the animal's time in the box before, during, and after the CS, it is best described as a contextual cue. Group $\mathbf{N}$ was placed in the compartment with the same body orientation but no food or food cup. Group P received three trials with the lid containing 30 pellets and three trials without the lid according to the following schedule: RNNRNR, NRNNRR, NNRRNR, RNRNRN, NNRNRR, RNNRNR, NRNNRR, and NNRRNR, where $\mathbf{N}$ represents a trial without food and $\mathbf{R}$, a trial with food. The amount eaten was recorded for all rats receiving food. By the end of the second day, each rat ate on every rewarded trial. The amount of Wayne Lab Blox was adjusted for the number of pellets eaten, so that each rat ate $14 \mathrm{~g}$ per day total.

Phase 3 started the day after Phase 2 ended. On Day 1 of Phase 3 the rats received eight trials, and for the other 5 days of the phase, the rats received six trials each day. The trials were the same as in Phase 1 except that shock no longer occurred and a 30-sec maximum for nonresponding was in effect. After $30 \mathrm{sec}$, the door closed, the clock and tone terminated, and the rat was removed to a holding cage for $20 \mathrm{sec}$ between trials.

\section{Results}

All analyses of variance included group and experimenter as between factors and trials and days as within factors. No differences involving experimenter were significant in any analysis, so this variable is not mentioned further.

There was no differences between groups in trials to meet the avoidance criterion [F $<1]$. Mean trials to criterion were: $26.6,25.6$, and 28.6 for Groups $R, N$, and $P$, respectively. On the last block of five criterion trials, the mean log latencies to respond were: $.345, .413$, and .407 for Groups $R, N$, and $P$, respectively $[F(2,24)=1.53]$.

On the first five trials in extinction, after counterconditioning and exposure in Phase 2, the mean log latencies were: $.574, .820$, and .809 for Groups $\mathbf{R}, \mathbf{N}$, and $\mathbf{P}$, respectively. As can be seen, all groups increased in latency from their acquisition level $[F(1,24)=189.37, p<.001]$. The size of this increase varied significantly by group $[F(2,24)=6.07$, p < $.01]$. Groups $\mathbf{N}$ and $\mathbf{P}$ increased in latency more than Group $\mathbf{R}$, although the increase in latency was significant for every group (Newman-Keuls tests, using the pooled error term as specified by Winer, 1971). 
In extinction testing, the same pattern of results occurred on each of the 6 days of testing [days $x$ group, $F<1$ ], although latencies did increase over days for all groups [days, $F(5,120)=3.76, p<.01$ ] Accordingly, mean latencies to response $\left[\log _{10}(1 \mathrm{a}-\right.$ tency +1$)$ ] are shown in Figure 1 summed over the 6 test days. Latencies were converted to logs to normalize the distribution for analysis of variance. The last two trials on Day 1 of Phase 3 were dropped from the analysis to equate the number of trials for each day of the analysis. As can be seen in Figure 1, Group $\mathbf{R}$ responded more rapidly than Group $\mathbf{N}$ on all trials. Group P began each day at the level of Group $\mathrm{N}$ and ended each day responding almost as rapidly as Group R. Analysis of variance showed that the difference associated with groups was not significant $[F(2,24)=2.90, p=.07]$, but there was a significant trials $\times$ groups interaction $[F(10,120)$ $=2.74, \mathrm{p}<.01]$. The significant trials $\times$ groups interaction justifies comparing groups on each trial (Winer, 1971). Subsequent Newman-Keuls tests (using the error term for groups pooled with the trials $\times$ groups error term, as specified by Winer, 1971) showed that Group $R$ responded more rapidly than Group $N$ on every trial except Trial 5. Group P responded significantly more slowly than Group $\mathbf{R}$ on Trials 1 and 2, but did not differ from Group $R$ on the remaining trials; Group $P$ was significantly faster than Group N on Trial 6, but did not differ from Group $N$ on the other trials ( $p s<.05$ ).

Differences between groups in percentage of successful "avoidances" in extinction (i.e., trials with latency less than $5 \mathrm{sec}$ ) were in the same direction as the latency differences. Mean percentages to avoid

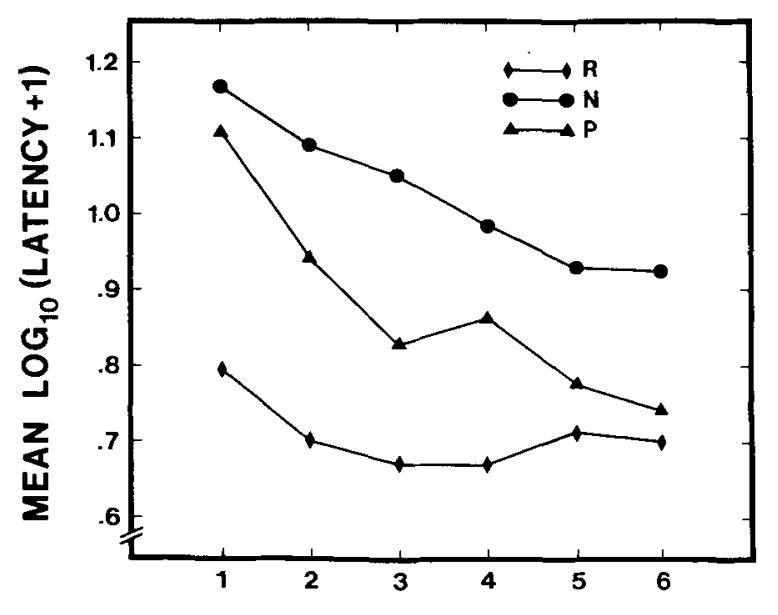

TRIALS

Figure 1. Mean [logio(latency +1$)]$ for each group on each trial of the Phase 3 extinction test summed over the 6 test days. Group $R$ was confined to the shock side with food in Phase 2, Group $N$ was confined without food, and Group $P$ was confined on half its trials in Phase 2 without food and half with food. for each group were: Group R, 86.57\%; Group N, $62.78 \%$; and Group P, $81.467 \%$. Differences between groups were significant $[F(2,24)=3.52$, $p<$ .05]. Subsequent Newman-Keuls tests showed that Group R "avoided" successfully more frequently than did Group N $(p<.05)$, while Group P did not differ significantly from either Group $\mathbf{R}$ or Group $\mathbf{N}$.

In counterconditioning, the mean number of pellets eaten per trial by Group P was 15.4 and that by Group R, 14.1.

\section{Discussion}

In Experiment 1, avoidance responding could have been produced by fear of the nominal $\mathrm{CS}^{+\mathrm{AV}}$ or of the context on the shock side, or both. Regardless of what cues elicited fear, the results of Experiment 1 show that giving food during exposure to these cues (context plus $\mathrm{CS}^{+\mathrm{AV}}$ ) can increase the resistance to extinction of an active avoidance response to that CS. The greater the number of food trials, the greater was the resistance to extinction. (The fewer the number of food trials, the less the resistance.) Either of the two factors discussed in the introduction could be responsible for these results. If removal of food from Phase 2 to Phase 3 produced frustration, which produced escape or which energized or directly elicited responses motivated by fear or associated with fear stimuli, then Group $\mathbf{R}$ should have responded fastest, followed by Group $P$, and then by Group $N$. The greater the number of food trials, the greater should be the expectancy of food and the greater should be the frustration when food is removed (Amsel, 1958). Group $R$ received 48 food trials, Group $P$ received 24 and Group $\mathbf{N}$ received none. A not incompatible hypothesis is that food is an important part of the stimulus context. Thus, for Group $R$, fear was conditioned with no food present; adding food to the context changes the context from that present in acquisition, so perhaps only generalized fear is extinguished. Also, fear is extinguished in the stimulus context of food. Then in testing food is removed, making the stimulus context like that in which fear was conditioned and unlike that in which fear was extinguished. Providing the food cue only during extinction would thus increase the fear present in the test for Group R. Group P experienced half of their CS exposures with food and half without food. Thus, only half of their trials were in the context used in the test (with no food). Group N, of course, had all their CS exposures without food, making them least resistant to extinction. Although the results of Experiment 1 do not distinguish between a frustration and a stimulus change interpretation, they do show that either or both of these processes are important in counterconditioning experiments, something that has not been demonstrated previously. 
The present results are not totally unprecedented. It is true that in previous experiments similar to Experiment 1 the counterconditioning group and a group exposed to the CS (groups analogous to Groups $\mathbf{R}$ and $\mathbf{N}$ ) usually have not differed significantly (Nelson, 1966; Wilson, 1973) when exposure to the CS is controlled. However, in Wilson's (1973) experiment, the counterconditioning group was more resistant to extinction than the exposed-only group, although not significantly so.

It should be pointed out that it is difficult to interpret the present results in terms of the conditioning of competing instrumental responses during the exposure phase. Group $\mathbf{R}$ received more reinforcements in the shock compartment than did Group P, which received more than Group N. Any staying response thereby conditioned should have been stronger in Group $R$ than in Group $P$, and stronger in Group $P$ than in Group N. This would produce less resistance to extinction in Group $\mathbf{R}$ than in Group $P$, and less in Group $P$ than in Group N, the reverse of the result obtained here.

\section{EXPERIMENT 2}

In Experiment 1, resistance to extinction of avoidance was less if confinement with the CS was without food (extinction, Group $N$ ) than if confinement was with food (counterconditioning, Group R). Performance of the partial counterconditioning group, confined with food and no food on different trials, was intermediate. Because the CS was given on all trials, the groups differed in the number of exposures to the shock side with food and with no food, as well as in whether the CS was associated with food, with no food, or with both food and no food. However, the number of food and nonfood trials on the shock side should not be important if either contextual control of fear to the CS or frustration elicited by the CS are responsible for the results. The important variable should be whether food is present or not when the CS is received. Experiment 2 was designed to determine if this was so.

In Experiment 2, all groups were trained to avoid shock using a tone CS and then confined to the shock side. The two critical groups received food on half the confinement trials and no food on the other half. On confinement trials, Group TN-R received the tone on its nonfood trials, Group N-TR received the tone on its food trials. Thus, for Group N-TR, the tone was associated with food, and for Group TN-R, it was not. Note that both groups received equal exposure to the tone. Both groups also received the same number of food and nonfood trials on the shock side. Therefore, any expectancy of food elicited by situational stimuli was equated across groups. And any counterconditioning of fear to the contextual place cues was equal for both groups. There were two additional groups: Group TN received only tone-nonfood trials, and Group TR received only tone-food trials.

If extinction of fear to the tone is specific to the context under which extinction is given, and food is part of the context, then Groups N-TR and TR should be more resistant to extinction than Groups TN-R and TN, because, for Groups N-TR and TR, the context is changed from extinction to test. During extinction, the CS is presented with food; in test, it is presented without food. Likewise, if presenting a tone that was previously associated with food and giving no food produces frustration and escape responses, Groups N-TR and TR should be more resistant to extinction than Groups TN-R and TN.

\section{Method}

Subjects. Forty naive rats of the same description as those used in Experiment 1 served as subjects.

Apparatus. The apparatus was the same as that used in Experiment 1.

Procedurs. Two experimenters ran the subjects, with each running half the rats in each group. The rats were assigned to groups after 14 days of ad-lib feeding. Handling, deprivation schedule, and the parameters of avoidance training were the same as in Experiment 1. Rats each received 20 pellets in their home cages on each of the 4 days prior to avoidance acquisition. The last rat finished avoidance acquisition in 12 days. For Group TR, the trials to criterion ranged from 15 to 56 , for Group TN from 15 to 53 , for Group N-TR from 15 to 50 , and for Group TN-R from 20 to 40 . When its turn in the running sequence occurred, once an animal finished avoidance training, it was picked up, handled briefly, and replaced in its home cage, as in Experiment 1. There was a 1-day break between Days 1 and 2 and a 2-day break between completion of avoidance acquisition for the last rat and the Phase 2 confinement trials.

In Phase 2, Groups TN-R and N-TR were run on each of 8 days, Groups TR and TN were run only on Days $2,4,6$, and 8 . On each day of Phase 2, Groups TN-R and N-TR received six confinement trials on the shock side (same procedures on confinement trials as in Experiment 1). For the first 4 days, there was no food given; for the last 4 days, 30 pellets in a Mason jar lid were given on each trial. For Group TN-R, the tone sounded on trials on the first 4 (nonfood) days; for Group N-TR, the tone sounded on trials on the last 4 (food) days. Group TN received 4 days of nonfood trials with the tone; Group TR received 4 days of food trials with the tone. Thus, all groups were equated for exposure to the tone.

In the Phase 3 test, there were 6 days of six trials each following the same test procedures as in Experiment 1.

\section{Results}

All analyses of variance included experimenter, tone on food vs. tone on nonfood trials, and consistent (TR + TN) vs. varied reward (TN-R + TR-N) on confinement trials as between factors and days and trials as within factors. In no analyses were any differences associated with experimenter significant, so this variable is not mentioned further.

There were no differences among groups in trials to meet the avoidance criterion $(\mathrm{Fs}<1)$ or in mean 
latencies to avoid on the last five trials (Fs $<1$ ). The mean numbers of trials to criterion were: Group TR, 16.5; Group TN, 15.3; Group N-TR, 16.2; and Group TN-R, 14.5. On the last five trials of acquisition, the mean latencies $\left[\log _{10}(\right.$ latency +1$\left.)\right]$ were: Group TR, .49; Group TN, .52; Group N-TR, .49; and Group TN-R, .51.

In extinction, Groups TR and N-TR performed similarly and both were more resistant to extinction than Groups TN and TN-R. The mean latencies to respond $\left[\log _{10}(\right.$ latency +1$\left.)\right]$ and the mean percentage of "avoidance" responses (trials with latencies less than $5 \mathrm{sec}$ ) for each group are shown in Table 1.

In analyses of latencies and percentage avoidances, the only significant between factor was whether the tone was presented on food or on nonfood trials. Groups that had the tone on food trials responded more rapidly $[F(1,32)=6.69, p<.02]$ and more frequently $[\mathrm{F}(1,40)=44.04, \mathrm{p}<.001]$ than rats that had the tone presented on nonfood trials. There were no interactions of the betweensubject variables with either days or trials.

The mean number of pellets eaten by groups given pellets on confinement trials were: Group TR, 9.28; Group TN-R, 10.69; and Group N-TR, 11.15.

\section{Discussion}

Experiment 2 demonstrated that the number of confinement trials on the shock side with food or no food is not critical in determining resistance to extinction to a CS. Rather, the critical factor is whether food or no food is given when the CS is present. If the CS was presented with food, stronger avoidance of the CS was shown in the test than if the CS was presented without food. Confinement trials given without the CS had no effect on resistance to extinction whether they were given with or without food. Group TN-R did not differ from Group TN in resistance to extinction, and Group N-TR did not differ from Group TR in resistance to extinction. Clearly, the important consideration is whether the CS is given with food or without food. And, surprisingly, the effects of giving food with the CS are the reverse of those expected from a process of counterconditioning-giving food with the CS produces greater resistance to extinction than giving no food.

Table 1

Performance of Each Group in Extinction

\begin{tabular}{lcc}
\hline Group & $\begin{array}{c}\text { Mean } \\
\log _{10} \text { of } \\
\text { (Latency }+1)\end{array}$ & $\begin{array}{l}\text { Percentage } \\
\text { "Avoidance" } \\
\text { Responses }\end{array}$ \\
\hline TR & .81 & 49.7 \\
N-TR & .77 & 57.5 \\
TN & 1.03 & 33.3 \\
TN-R & .99 & 36.4 \\
\hline
\end{tabular}

As indicated above, this finding can be explained on the basis of contextual control of fear extinction (Bouton \& Bolles, 1979), with food being the contextual cue, or on the basis of frustration produced by how, in the test, receiving without food a cue that had previously been associated with food. These hypotheses are not incompatible, and it seems entirely likely that both processes operate to increase resistance to extinction in counterconditioning groups in the one-way avoidance situation. The evidence that frustration can motivate escape is strong (e.g., Daly, 1974), and the conditions in counterconditiong experiments using the one-way active avoidance situation are those shown in many experiments to produce frustration. However, there is no direct evidence that food can function as a contextual cue in controlling fear and fear reduction. The purpose of Experiment 3 was to provide direct evidence for the hypothesis that food can function as a contextual cue in controlling fear and fear reduction in counterconditioning experiments.

\section{EXPERIMENT 3}

The usual counterconditioning group is given food during counterconditioning, yet food is not present in fear conditioning or in the test for residual fear. This contextual difference could be the basis for a discrimination: when there is no food, shock is given; when there is food, no shock is given. In Experiment 3, this hypothesis was evaluated by having food present for some groups during fear training. Because this procedure is difficult to implement in avoidance conditioning, conditioned suppression of licking was used as a measure of fear in Experiment 3.

Groups received fear conditioning with or without food present, then received counterconditioning or extinction, and then were tested for residual fear with no food present. All groups were tested with no food present because the presence of a dish of food in the test would interfere with the lick response being measured. Because the test was without food, giving food during fear reduction should be less effective than not giving food then. So groups given extinction should show less fear in the test than groups given counterconditioning (the more similar test conditions are to the no-shock phase, the less fear should be shown), as found in Experiments 1 and 2. In contrast, giving food during fear conditioning should have the opposite effect. Because the test was given without food, groups that had had fear conditioning without food should show greater fear than those that had had fear conditioning with food (the more similar to the shock phase the test conditions are, the greater the amount of fear shown should be). Thus, the main predictions are that rats given food during fear conditioning should show less fear in the final fear test than those not given food, 
while rats given food during the fear-reduction phase should show more fear in the final fear test.

\section{Method}

Subjects. Twenty-four male albino rats of the same description as Experiments 1 and 2 were used.

Apparatus. The apparatus consisted of two identical modified Scientific Prototype (Model H-110) operant chambers $(23.5 \times$ $20.3 \times 19 \mathrm{~cm}$ ) with bronze-colored aluminum end panels and clear Plexiglas sides and covers. The floors consisted of 16 parallel grid bars $(.4 \mathrm{~cm}$ diam, $1.5 \mathrm{~cm}$ on center). A drinking tube projected $1.5 \mathrm{~cm}$ into the chamber through the lower right of the front end panel, $3 \mathrm{~cm}$ from the right side panel, and $2.25 \mathrm{~cm}$ from the floor. A removable, aluminum tube guard was attached to the front panel around the tube so that only the tip of the tube was exposed through a 1.4-cm-diam hole in the guard face. The drinking tube and the first eight grids nearest the front wall were attached to a Lafayette Instrument Co. drinkometer (Model 58008 ), which registered each lick made by the rat. Shock could be delivered to the grid floor via two Lafayette Instrument Co. shockers (Model 83404). Constant illumination was produced by a 6-W shieided light located in the center of the front panel $2.5 \mathrm{~cm}$ from the top. Each operant chamber was enclosed in a Scientific Prototype sound-attenuating chamber (Model SPC-300, $63.5 \times$ $34.3 \times 39.4 \mathrm{~cm}$ ). Background noise was produced by the exhaust fans of the sound chamber.

Procedure. The study was run by two experimenters, each running half the rats in each group. Following 5 days of ad-lib feeding (Day 1), rats were assigned randomly to four groups of six rats each: FF, FN, NF, NN. The first letter of the group name indicates whether the rats were given food during shock experience (F) or no food during shock experience (N). The second letter refers to whether food was present during fear extinction (F) or not $(\mathrm{N})$. Half the animals in each group were assigned to each of the operant chambers. Each rat used only one chamber throughout the study.

On Day 2, the water bottles were removed from the home cages of all rats. They were given $20 \mathrm{ml}$ of water on Day 3. Adlib water was reinstated on Day 4, and all food was removed from the rats' cages. Beginning on Day 5, all rats were maintained on ad-lib water and $14 \mathrm{~g}$ of lab chow daily (amount of food given in the home cage daily was always adjusted for any food eaten in the apparatus). On Day 7, all rats received $20.045-\mathrm{g}$ Noyes pellets in a Mason jar lid that was attached to the floor of the home cage. If the rat ate the food within $10 \mathrm{~min}$, the dish was removed from the cage. If any rat failed to eat all the pellets in the $10 \mathrm{~min}$, the dish remained in the cage overnight and the remainder of the rat's daily ration was added.

On Day 8, each rat was placed in its operant chamber for 5 min with Mason jar lid containing 20 pellets. The amount eaten during the 5 min was recorded. The rats were run randomly in squads of four, consisting of one rat from each group, with Squad 1 always going prior to Squad 2, etc. Fifteen minutes after their last trials, the rats in each squad were fed their daily rations.

Lick training began on Day 9. All rats were trained to lick for an $8 \%$ sucrose solution in the operant chambers. On the first trial of training, the tube guards were removed from the chambers to insure that the rats would find the drinking tubes. On this trial, for which no data were recorded, each rat remained in the chamber 2 min after it made its first lick response. On all trials during lick training, the rats were placed in the chamber facing away from the tube. Beginning with Trial 2, the tube guard was replaced and the rats remained in the chamber until they had completed 50 licks or $10 \mathrm{~min}$ had passed, whichever came first. Training continued for 11 trials with the guard on. Latency to 50 licks (in .01 sec) was recorded on each trial. All other procedures were the same as for placements. Lick training spanned 4 days.

On Days 13 and 14, all rats received $121.0-\mathrm{mA}$, .5-sec shocks on a VI 40-sec schedule, with the ITI varying from 30 to $50 \mathrm{sec}$.
Two rats were run at a time, keeping as close to squads as possible. Groups FF and FN were placed in the chamber with a Mason jar lid (not attached to the floor) containing 40 Noyes pellets. Because the jar lid was metal on the outside, had the rats entered the small space between the lid and the wall of the chamber and stood on the same grids as the lid, they would have received no shock. However, since they did not do this, but instead remained in the other part of the chamber, they received a slightly higher intensity of shock than did the groups without the jar lid (at most .125 mA more). This higher shock intensity should produce, if anything, greater fear for groups with food present during fear conditioning, which is contrary to the result predicted. Groups NN and NF were placed in the chamber with no jar lid present. All rats were placed facing away from the lick tube area with the tubes and the grid leads to the drinkometers removed from the chamber. All other procedures were the same as for lick training.

Extinction was given on Days 15 and 16. On these days, each rat was placed in the chamber for $20 \mathrm{~min}$ with no shocks. Groups FF and NF were placed there with the jar containing 40 pellets. Groups NN and FN were placed without the jar lid. All other procedures were the same as in shock training.

On Day 17, the drinking tubes were replaced in the chambers and all rats were tested for latency to complete 50 licks with no jar lid present in the chamber. The rats had a maximum of $1 \mathrm{~h}$ in which to complete the 50 licks. All other procedures were the same as in lick training.

\section{Results}

The mean $\log$ latency $\left[\log _{10}(\right.$ latency +1$\left.)\right]$ to complete 50 licks for each group on the last training trial and on the test trial are shown in Table 2. Data were analyzed in factorial analyses of variance, including food vs. no food in fear conditioning, food vs. no food in fear extinction, and experimenter as factors. No differences associated with the experimenter were significant, so this variable is not mentioned further. Analysis on the test trial showed that groups that received fear conditioning with no food present $(\mathrm{NF}$ and $\mathrm{NN}$, mean $=2.37$ ) were more afraid in the no-food test than were groups given fear conditioning with food (FF and FN, mean $=2.08$ ) $[F(1,16)$ $=6.12, \mathrm{p}<.03$ ]. In contrast, groups given extinction (FN and NN, mean $=1.98$ ) showed less fear on the test than those given counterconditioning (FF and $\mathrm{NF}$, mean $=2.97)[F(1,16)=16.83, p<.01]$. The interaction of these variables was not significant $(F<1)$. A similar analysis on the last training trial showed no significant differences. Groups to be given fear conditioning with food did not differ significantly on the last day of lick training (mean = 1.42) from those to be given training without food (mean $=1.12)[F(1,16)=2.81, p<.10]$ (mean square error was larger during lick training than during test-

Table 2

Mean $\log _{10}($ Latency +1$)$ to Complete 50 Licks

\begin{tabular}{ccc}
\hline Group & Training & Test \\
\hline IF: & 1.47 & 2.37 \\
IN & 1.37 & 1.79 \\
NN & 1.14 & 2.17 \\
NF & 1.10 & 2.58 \\
\hline
\end{tabular}


ing, accounting for the fact that this size difference was not significant in training while slightly larger differences were significant in testing). The differences between groups to be given food or not in fear extinction produced $F<1$ on the last day of lick training, as did the interaction of these variables.

A third analysis of variance was done comparing latencies on the last lick training day to differences on the test day. In this analysis, no differences associated with experimenter were significant. Differences associated with food vs. no food in fear conditioning were significantly larger on the test trial than they had been in lick training [food vs. no food in fear conditioning $\times$ training vs. test, $F(1,16)$ $=14.72, \mathrm{p}<.01]$. Also, differences associated with receiving food vs. no food in fear extinction were significantly larger on the test trial than they had been in lick training (food vs. no food in extinction $\times$ training vs. test $[F(1,16)=8.78, p<.01]$. Thus, differences in test were significantly greater than any preexisting training differences.

For groups given pellets in fear conditioning (FF and $F N$ ), the number of pellets consumed decreased from Day 1 to Day 2 of conditioning. On Day 1, the mean number consumed was 28.4, and on Day 2, it was 15.24. In extinction, Group FF increased the number of pellets eaten from 16.6 on Day 1 to 28.4 on Day 2. Group NF ate all 40 pellets on both days of extinction.

\section{Discussion}

The results of Experiment 3 show that the presence of food pellets is an important part of the context controlling both fear conditioning and fear reduction. In accordance with the assumption that the presence vs. absence of food pellets is an important part of the stimulus complex controlling fear and fear reduction, in a test with no food pellets, ${ }^{1}$ groups that had food pellets in fear conditioning showed less fear than groups given fear conditioning without food pellets. The stimulus complex in the test is more similar to that present in conditioning for groups given fear conditioning with no food pellets, which accounts for their greater fear.

Also, as expected, if the presence vs. absence of food pellets is an important part of the stimulus complex controlling fear and fear reduction, in a test with no food pellets, groups given fear extinction without food pellets showed less fear than groups given fear extinction with food pellets. This latter effect is relevant to counterconditioning experiments. In counterconditioning experiments, training and testing is customarily given with no food. Thus, the counterconditioning group given food in the fear extinction phase should show less fear in the test because of the removal of the food cue from extinction to test. It is also possible that, for a counterconditioning group, adding food in extinction when it was not present in fear conditioning may reduce the degree of fear elicited during extinction (because of stimulus change), contributing to the residual fear present in a subsequent test. It appears that one reason counterconditioning experiments can fail to find counterconditioning is the contextual control of fear, with food being the contextual cue of importance.

\section{GENERAL DISCUSSION}

In all three experiments, groups given food during fear extinction subsequently showed greater residual fear than those not given food. This is the reverse of what would be expected on the basis of a counterconditioning process. We have suggested that the results of Experiments 1 and 2 can be attributed in part to the aversive effects of removing food (frustration), which motivates escape responses in the test phase of the avoidance situation, and/or to the stimulus effects of food cues. For a counterconditioning group, fear acquisition is conducted without food, extinction is given with food, and then testing is given without food. This stimulus difference provides the basis for a discrimination-when food is present, the situation and the CS are safe. When food is not present, shock is given. Experiment 3 showed that food can, indeed, function in this manner. In Experiment 3, in a test with no food, having no food present during conditioning increased the amount of fear shown, whereas having no food present during fear extinction decreased fear shown.

Although we have suggested that the operation of frustration and stimulus change can interfere with measuring a counterconditioning process, there was no evidence at all for a counterconditioning process in these experiments. In all three experiments, extinction was more effective in reducing fearmotivated behavior than was counterconditioning. While these results can be explained in terms of stimulus change, the fact that the results were the opposite of those expected on the basis of counterconditioning suggests that if there is a counterconditioning process, it is weak, weaker than stimulus change effects. Because the empirical evidence for a counterconditioning process is weak, it seems worthwhile to consider the theoretical basis for expecting a counterconditioning effect.

The first conception of counterconditioning was originally suggested by Guthrie (1938) and adopted by Wolpe (1958). Guthrie suggested that for an association between a stimulus and a response to be broken, the stimulus must be associated with a different response. Wolpe's analysis of how anxiety is reduced in counterconditioning was also in terms of competing responses. He suggested that, during original fear conditioning, a fear response is conditioned to the CS $\left(S-R_{1}\right)$, and during countercon- 
ditioning an incompatible response is conditioned to the CS $\left(S-R_{2}\right)$, thereby breaking the connection between $S$ and $R_{1}$. There is good evidence that if incompatible responses are involved in counterconditioning, they are not necessarily peripheral CRs. Counterconditioning procedures can produce a weakening of a peripheral $R_{1}$ and a strengthening of a peripheral $\mathbf{R}_{\mathbf{2}}$ even if the peripheral responses are not incompatible. Scavio (1974), for example, showed that following conditioning of the nictitating membrane response to a CS by CS-shock pairings, associating that CS with water decreased the frequency of nictitating membrane responses while the number of jaw movement CRs increased, even though the jaw movement and nictitating membrane responses are not incompatible.

Therefore, recently a common view has been that the interference produced by counterconditioning is not between peripheral responses but between central motivational states. Counterconditioning has been viewed as just one example of a more general reciprocal inhibition of motivational states. A common assumption is that there are two central motivational states, appetitive and aversive, with a reciprocally inhibiting influence on one another (e.g., Bindra, 1974; Estes, 1969; Konorski, 1967; Millenson, 1971; Rescorla \& Solomon, 1967). In a counterconditioning procedure, providing food excites the appetitive motivational system, thereby weakening the aversive motivational system. The evidence for this idea is weak at best. Lovibond and Dickinson (1982), for example, conditioned a nictitating membrane response by CS-shock pairings and then associated that CS with both shock and sucrose on different trials. The development of a jaw-movement CR did not reduce the frequency of the eyeblink response as would be expected on the basis of counterconditioning, but actually enhanced the eyeblink somewhat.

Lovibond and Dickinson (1982) suggest one hypothesis that would make these results compatible with the general idea that appetitive and aversive motivational systems reciprocally inhibit one another, namely that a CS for sucrose inhibits, rather than excites, appetitive motivation. This accounts for CSsucrose pairings' actually enhancing the eyeblink CR: the reduction of appetitive motivation would enhance the action of the aversive motivational system. Scavio and Gormezano (1980) recently made the same suggestion to account for their results, which showed that prior conditioning of a jaw movement $\mathrm{CR}$ to a $\mathrm{CS}$ as a result of CS-water pairings facilitated subsequent nictitating membrane conditioning to that CS. This suggestion implies that counterconditioning procedures should be less effective than extinction procedures in reducing fear, as we found here. Food reduces appetitive motivation, which should thereby enhance the action of the aversive motivational system; this means there is no counterconditioning process whereby fear is weakened through providing food. This hypothesis, unsupplemented by other processes such as stimulus change, has difficulty explaining cases in which counterconditioning procedures appear to be more effective than extinction procedures in reducing fear (e.g., Wilson \& Dinsmoor, 1970). The variety of results that can occur when an aversive CS is associated with an appetitive US suggests that an alternative conceptualization of counterconditioning may be desirable.

One alternative that can potentially accommodate a variety of results is that the interference in counterconditioning procedures is central but not between motivational states. Rather, the interference may be between different expectations. In counterconditioning procedures, for example, the animal may learn that the CS means shock in Phase 1 and then food in Phase 2. This learning will be apparent if the CR appropriate to the shock decreases and the CR appropriate to food increases during Phase 2 (as shown by Scavio, 1974). This does not necessarily mean, however, that the animal has forgotten or unlearned that the tone meant shock. Counterconditioning procedures can be viewed as one subset of procedures in which cues are associated with two different events. Thus, the same rules should apply to counterconditioning as apply in A-B, A-C transfer paradigms in general. Interestingly, in human work, while the A-B, A-C paradigm was originally thought to produce unlearning of the $A-B$ association, subsequent work has been more consistent with the idea that both A-B and A-C are stored and performance in a test will reflect how many retrieval cues at test are appropriate to A-B as opposed to A-C (Crowder, 1976). When a CS is paired with shock and then with food, if the presence vs. absence of food functions as a retrieval cue, then, in a test without food, it would be expected that greater fear would be shown if no food, rather than food, had been given during fear conditioning, because the absence of food would retrieve the memory of shock, and thereby fear. Likewise, in the test without food, less fear should be shown if no food rather, had been given during fear extinction, because the absence of food would retrieve the memory of no shock. These, of course, were the results in Experiment 3. This interpretation is also consistent with Wilson and Dinsmoor's (1970) results. These investigators found that, after training rats to stay on a platform to avoid shock, a group given food on the platform (counterconditioning) left the platform sooner than a group not given food. Because fear conditioning was given without food, the situation in test was less similar to that in which fear was conditioned for the counterconditioning group than for the other group, 
which could account for their lesser fear. (As mentioned earlier, frustration could also have played a role for the animals in this group if they ran out of food in the test.)

Another example of an A-B, A-C paradigm is extinction of fear to a CS. Bouton and Bolles (in press) recently reviewed research showing that contextual control of fear is strong when a CS has been subjected first to acquisition then extinction. They suggest that this is because the CS is ambiguous, having been associated with shock and with no shock, and that the animal thus relies on the context for what to expect. This view is highly similar to the present analysis of counterconditioning. Both analyses suggest that in A-B, A-C paradigms the A-B association is not necessarily broken, and performance following A-B, A-C training is determined by the extent to which the cues present reinstate or elicit the A-B association as opposed to the A-C association.

\section{REFERENCES}

Amset, A. The role of frustrative nonreward in noncontinuous reward situations. Psychological Bulletin, 1958, 55, 102-119.

Azrin, N., \& Hake, D. F. Positive conditioned suppression: Conditioned suppression using positive reinforcers as the unconditioned stimuli. Journal of the Experimental Analysis of Behavior, 1969, 12, 167-173.

Bindra, D. A motivational view of learning, performance and behavior modification. Psychological Review, 1974, 81, 199-213.

Bouton, M. E., \& Bolles, R. C. Contextual control of the extinction of conditioned fear. Learning and Motivation, 1979, $10,445-466$.

Bouton, M. E., \& Bolles, R. C. Contexts, event-memories, and extinction. In P. Balsam \& A. Tomie (Eds.), Context and learning. Hillsdale, N.J: Erlbaum, in press.

Brown, R. T., \& WAGNer, A. R. Resistance to punishment and extinction following training with shock or nonreinforcement. Journal of Experimental Psychology, 1964, 68, 503-507.

Crowder, R. G. Principles of learning and memory. Hillsdale, N.J: Erlbaum, 1976.

DALY, H. B. Reinforcing properties of escape from frustration aroused in various learning situations. In O. H. Bower (Ed.), The psychology of learning and motivation. New York: Acrdemic Press, 1974.

Delphato, D. J., \& Jackson, D. E. Counterconditioning and exposure-only in the treatment of specific (conditioned suppression) and generalized fear in rats. Behaviour Research and Therapy, 1973, 11, 453-461.

Dickinson, A., \& Pearce, J. M. Inhibitory interactions between appetitive and aversive stimuli. Psychological Bulletin, 1977, 84, 690-711.

Estes, W. K. Outline of a theory of punishment. In B. A. Campbell \& R. M. Church (Eds.), Punishment and aversive behavior. New York: Appleton-Century-Crofts, 1969.

Guthrie, E. R. The psychology of human conflict. New York: Harper \& Row, 1938.

JonEs, M. C. The elimination of children's fears. Journal of Experimental Psychology, 1924, 7, 382-390.

KAzoIN, A. E., \& WILson, G. T. Evaluation of behavior therapy: Issues, evidence, and research strategies. Cambridge, Mass: Ballinger, 1978.

KLEIN, B. Counterconditioning and fear reduction in the rat. Psychonomic Science, 1969, 17, 150-151.

KONORSKI, J. Integrative activity of the brain: An interdisciplinary approach. Chicago: University of Chicago Press, 1967.
Lovibond, P. F., \& Dickinson, A. Counterconditioning of appetitive and defensive CRs in rabbits. Quarterly Journal of Experimental Psychology, 1982, 34, 115-126.

MCAlliste R, W. R., \& MCAlliste R, D. E. Behavioral measurement of conditioned fear. In F. R. Brush (Ed.), A versive conditioning and learning. New York: Academic Press, 1971.

Millenson, J. R. A motivational-reinforcement theory of emotion. Studia Psychologia, 1971, 13, 222-238.

NELson, F. Effects of two counterconditioning procedures on the extinction of fear. Journal of Comparative and Physiological Psychology, 1966, 62, 208-213.

Pavlov, I. P. Conditioned reflexes. Oxford, England: Oxford University Press, 1927.

Poppen, R. Counterconditioning of conditioned suppression in rats. Psychological Reports, 1970, 27, 659-671.

RE1D, L. D. Processes of fear reduction in systematic desensitization: An addendum to Wilson and Davison (1971). Psychological Bulletin, 1973, 79, 107-109.

Rescorla, R. A., \& Solomon, R. L. Two-process learning theory: Relationships between Pavlovian conditioning and instrumental learning. Psychological Review, 1967, 74, 151-182.

Scavio, M. J., Jn. Classical-classical transfer: Effects of prior aversive conditioning upon appetitive conditioning in rabbits. Journal of Comparative and Physiological Psychology, 1974, 86, 107-115.

Scavio, M. J., Jr., \& Gormezano, I. Classical-classical transfer: Effects of prior appetitive conditioning in rabbits. Animal Learning \& Behavior, 1980, 8, 218-224.

Sermat, V., \& Shephand, A. H. The effect of a feeding procedure on persistent avoidance responses in rats. Journal of Comparative and Physiological Psychology, 1959, 52, 206-211.

Wilson, E. H., \& Dinsmoor, J. A. Effect of feeding on "fear" as measured by passive avoidance in rats. Journal of Comparative and Physiological Psychology, 1970, 70, 431-436.

WILSON, G. T. Counterconditioning versus forced exposure in extinction of avoidance responding and conditioned fear in rats. Journal of Comparative and Physiological Psychology, 1973, 82, $105-114$.

Wilson, G. T., \& Davison, G. C. Processes of fear reduction in systematic desensitization: Animal studies. Psychological Bulletin, 1971, 76, 1-14.

WiNEn, B. J. Statistical principles in experimental design (2nd ed). New York: McGraw-Hill, 1971.

Wolpe, J. Psychotherapy by reciprocal inhibition. Stanford: Stanford University Press, 1958.

\section{NOTES}

1. Scavio (1974) reported that a conditioned nictitating membrane response, formed with CS-shock pairings, weakened more rapidly when the CS was subsequently associated with water than when the CS was presented alone. However a group for which the CS and water were presented unpaired also showed rapid extinction of the nictitating membrane response, as rapid as the group for which the CS and water were paired. Thus, the study does not demonstrate a clear counterconditioning effect produced by CS-water pairings.

2. It could be suggested that because sucrose is available in the test, the test is best described as with food. However, the food cue being manipulated, and thus the one of importance, is that characteristic of food pellets. The groups did not differ in presence vs. absence of sucrose in any phase of the experiment. No group had sucrose available in either fear conditioning or fear extinction, and all groups had the tube with sucrose available in test. Thus, differences between groups cannot be attributed to the cue provided by sucrose.

(Manuscript received September 22, 1982; revision accepted for publication March 11, 1983.) 